D.O.I.: $10.3895 /$ gi.v12n4.4706

\title{
ANÁLISE DA AUTOMAÇÃO DE TERMINAIS PORTUÁRIOS DE CONTÊINERES
}

\section{ANALYSIS OF AUTOMATION OF PORT TERMINALS OF CONTAINERS}

\author{
Delmo Alves de Moura ${ }^{1}$; Marcelo Patrícioº ${ }^{2}$ Rui Carlos Botter ${ }^{3}$ \\ ${ }^{1}$ Universidade Federal do ABC - UFABC - São Paulo - Brasil \\ delmo.moura@ufabc.edu.br \\ ${ }^{2}$ Brasil Terminal Portuário - Santos/SP - Brasil \\ m.patricio@btp.com.br \\ ${ }^{3}$ Universidade de São Paulo - USP - São Paulo - Brasil \\ rcbotter@usp.br
}

\begin{abstract}
Resumo
Este trabalho faz uma análise dos principais terminais portuários brasileiro, especialmente no porto de Santos, em São Paulo, relacionado com automação das operações portuárias na movimentação de contêineres. O trabalho foi realizado através de pesquisa em campo para levantamento da situação atual dos terminais em Santos visando descrever o grau de automação na movimentação, transbordo e armazenagem de contêineres. A pesquisa foi realizada através de um questionário em campo e se realizou uma revisão sobre o tema, assim como utilizou-se de benchmarking internacional para se ter parâmetro de automação portuária internacional. Buscouse fazer uma varredura na literatura que aborda o tema para se fundamentar teoricamente. $O$ artigo relata os principais itens para se levado em consideração no processo de automação de terminais portuários, tomando como referência a capacidade de transporte dos navios em TEUs (contêineres de vinte polegadas). A relação entre os tamanhos dos navios (capacidade de transporte) dos equipamentos encontrados nos terminais para realizar as operações de carregamento de descarregamento, com maior eficiência e eficácia operacional.
\end{abstract}

Palavras-chave: capacidade dos navios, automação portuária, movimentação contêineres.

\section{Introdução}

Este trabalho trata da análise de equipamentos de movimentação em terminais portuários, em especial na automação de movimentação de contêineres, no porto de Santos e outros terminais no Brasil. Dessa forma, apresenta-se uma visão sistêmica de terminais e das suas principais características técnicas.

Como referência de terminais automatizados, os dois terminais considerados os mais automatizados em operação na Europa são: o Container Terminal Altenwerder (CTA) em Hamburgo na Alemanha do operador HHLA (Hamburger Hafen und LogistikAG) e o Euromax 
Terminal em Roterdã na Holanda do operador ECT (Europe Container Terminals) que pertence ao grupo HPH (Hutchison Port Holdings).

O terminal de Maasvlakte 2, em Roterdã, é considerado o maior projeto de engenharia civil na Holanda; trata-se da construção de um novo porto e de infraestrutura de apoio em terra recuperada ao lado do terminal de Maasvlakte; A área total é de aproximadamente 167 hectares, com 2.800 metros de cais e outros 500 metros adicionais para operação de barcaças, com capacidade para 2,7 milhões de TEUs, os primeiros portêineres operados por meio de controle remoto da empresa Cargotec, guindastes para o terminal de barcaça, guindastes automatizados de pátio sobre trilhos (RMGs) da Kuenz, Guindastes para atendimentos dos ramais ferroviários na linha de cais, veículos guiados automatizados (AGVs) com bateria de elevação da Gottwald, estações robóticas de trocas de bateria da Gottwald, desenvolvimento conjunto dos Sistemas de Gerenciamento e Planejamento de Operações dos Terminais (TOS) e do Sistema de Controle dos Equipamentos (ECS) entre Navis (Cargotec) e TBA (Gottwald).

As construções do terminal são realizadas conforme as regras de certificação de desempenho ambiental da Building Research Establishment Environmental Assessment Method (BREEAM) adotadas pelo Dutch Green Building Council. Outra item sustentável do terminal está relacionado a uso de eletrificação de todo os equipamentos, permitindo que o terminal seja livre de emissão de $\mathrm{CO}_{2}, \mathrm{NO}_{\mathrm{x}}$, assim como de outras partículas. Como referência, a partir de 2009, o terminal de Maasvlakte I se tornou o primeiro terminal de contêineres do mundo alimentado por energia eólica.

\section{EVOLUÇÃO E PERSPECTIVAS DA MOVIMENTAÇÃO DE CONTÊINERES}

Não se sabe exatamente o número de contêineres existente no mundo atualmente, mas, de acordo com World Container Census 2012 da Drewry Maritime Container Research, a frota mundial de contêineres cresceu mais de 400\% nos últimos 20 anos, sendo 5,3\% em 2012, o que adiciona mais 1,6 milhões de TEU a um número estimado de 32,9 milhões de unidades.

Davidson (2014) indica o tamanho da indústria de contêiner global com uma movimentação total de contêineres de aproximadamente 640 milhões de TEUs/ano, mais de 5.000 portêineres ao redor do mundo e cerca de 600 quilômetros de berços, em 700 terminais especializados na movimentação de contêineres e mais outros 600 terminais multi-propósito (multi-purpose) e instalações para operação Roll on Roll off (RO-RO). A estimativa de receita é de US\$ 48 bilhões e EBITDA de US\$ 11 bilhões (aproximadamente 22\%).

De acordo com a publicação World Top Container Ports 2013 da Container Management, apenas os 120 primeiros portos no mundo movimentaram em 2012 o volume de aproximadamente 514,2 milhões de TEUs (Twenty Equivalent Units ou Unidade Equivalente de 20”), uma variação de 
21,5 milhões de TEUs e 4,38\% superior a 2011. Como referência, apenas os cinco primeiros portos do mundo (Shangai, Cingapura, Hong Kong, Shenzhen e Busan) movimentaram 127,2 milhões de TEUS.

O crescimento médio do volume de contêineres no Brasil ao ano foi de 10,1\%, mesmo considerando os anos de desaceleração pela crise financeira de 2008 e 2009; descartando-se apenas o ano de 2009, esse indicador sobe para 12,0\%. O crescimento anualizado da movimentação somado supera $150 \%$ nos últimos 13 anos; no mesmo período de acordo com a Associação Brasileira dos Terminais de Contêineres de Uso Público (ABRATEC) foram realizados investimentos em obras civis, aquisição de equipamentos de movimentação e treinamento e especialização da mão de obra da ordem de US\$ 2,8 bilhões. Adicionando os investimentos realizados pelos terminais da EMBRAPORT e BTP que não são associadas à ABRATEC, esse número se aproxima de US\$ 5 bilhões.

Entretanto, é emblemático o crescimento da movimentação nos diversos portos em praticamente uma década, os portos de Rio Grande e Rio de Janeiro dobraram a sua movimentação, os portos de Santos, Paranaguá, Salvador, Vitória e São Francisco do Sul (adicionada à movimentação de Itapoá) tiveram as suas movimentações triplicadas; enquanto Suape, Manaus e Itajaí (somado o volume de Navegantes, do terminal da Portonave) sextuplicaram seus volumes. E há casos especiais como Pecém e Itaguaí (antigo porto de Sepetiba) que praticamente não existiam 10 anos atrás e operações incipiente em Vila do Conde e Imbituba.

A relação entre os contêineres de 40" (12 metros) e de 20" (6 metros), o chamado fator TEU tem sido historicamente de 1,5. No ano de 2012 foram movimentados 5,47 milhões de unidades que multiplicadas por 1,5 atingem os 8,2 milhões de TEUs. Esse fator varia conforme o tráfego ou serviço, por exemplo: os navios que escalam na costa oeste americana e Extremo Oriente possuem uma parcela maior em contêineres de 40", ou no caso dos contêineres do tipo refrigerado, os quais são na sua totalidade são de 12 metros. Em 2013, a movimentação atingiu 5,8 milhões de unidades, o equivalente a 8,7 milhões de TEUs.

Sobre a infraestrutura implantada para conteinerização no Brasil, Robinson (1985) afirmou que essa nascera inadequada e deficiente para atendimento das exigências dos armadores. Passadas quase três décadas, Salgado (2012) discorreu no $1^{\text {o }}$ fórum de contêineres sobre a questão da adequação permanente dos terminais e demonstra como a atividade portuária é de capital-intensiva e que a infraestrutura e equipamentos ensejam altos custos fixos. Ele apresentou os seguintes desafios:

- Economias de escala, por meio das quais o custo unitário será reduzido na proporção do número de contêineres movimentados, amortizando os altos custos fixos num volume maior de contêineres movimentados; 
- Economias de escopo, isto é, o custo de produção unitário será reduzido quando o serviço é prestado em conjunto com outro, de forma que a oferta de um mitigue o custo do outro (trade-offs ou compensação de custos);

- Economias de densidade, por meio de ganhos econômicos decorrentes da agregação de consumidores vis à vis ao fator tempo. Na atividade portuária, há um ganho crescente de produtividade verificado quanto maior for o número de berços de um mesmo terminal, pois menor será a ociosidade de cada berço e mais fácil será a distribuição, no tempo, dos navios por berço.

Além desses desafios, Salgado (2012) apresentou parâmetros físicos que determinam a capacidade de um terminal de contêineres, a saber: Capacidade de atendimento dos gates, capacidade de pátio e capacidade de operações de carga e descarga dos berços”.

Esses parâmetros de capacidade citados anteriormente são os mesmos que compõem uma estrutura clássica ou tradicional de terminal, retratados por diversos autores, como Casaca (2005) que descreve uma estrutura abrangente de terminal portuária como subsistemas de portões (Gates) de acesso rodoviário e ferroviário, pátios de armazenagem e berços ou Steenken; Vo $\beta$ e Stahlbock (2004), que de forma mais detalhada define um terminal de contêineres como um sistema aberto identificado por meio de três subsistemas ou áreas de operações (área de operações intermodal de caminhões e vagões, área de armazenagem de contêineres e área de operações de navios), e com duas interfaces externas, sendo a primeira a interface de operações de costado com as operações de embarque e descarga dos navios e, a segunda, uma interface das operações de retaguarda de recebimento e entrega de contêineres de/para caminhões e vagões (CHOE, KIM, RYU, 2016)

Peixoto (2005) apresenta um modelo de caracterização sistêmica de um terminal de contêineres, formado por cinco subsistemas, a saber:

- Subsistema Costado dedicado à chegada e à atracação de navios;

- Subsistema Operações de descarga e embarque de contêineres dos navios;

- Subsistema Fluxo de transferência de contêineres entre o costado e pátio (usualmente chamado de "retaguarda");

- Subsistema Armazenagem para recebimento e posicionamento dos contêineres; e,

- Subsistema Transbordo (Gates) responsável pela intermodalidade do transporte.

\section{EVOLUÇÃO DOS NAVIOS PORTA-CONTÊINERES}

Os navios porta-contêineres evoluíram rapidamente desde a primeira viagem entre Nova Iorque e Houston do navio tanque convertido Ideal X em 1956, com $135 \mathrm{~m}$ de comprimento e capacidade para 500 TEUs, até a viagem inaugural do primeiro navio da série "Triple-E" do armador dinamarquês Maesk Lines, o M.V Maersk Kinney Moller em agosto de 2013, com 400 
metros de comprimento ( $L O A)$ e 59 metros de largura ou Boca (Breadth) e com capacidade para 18.200 TEUs (sendo 10.600 TEUs nos conveses e 7.600 TEUs nos porões), 23 colunas (Rows) e 10 Alturas (Tiers) no convés.

A propósito, o nome Triple-E é derivado de três princípios: Economia de escala, Eficiência Energética, Eficiência Ambiental (E्Economy of scale, Énergy efficient and $\underline{E}$ nvironmentally improved); essa classe de navio vem sendo construída pelo estaleiro da Daewoo Shipbuilding and Marine Engineering (DSME) na Coreia do Sul, com custo de US\$ 190 milhões por navio; e o que comprova esses princípios são as comparações de indicadores como: a redução de emissão de $\mathrm{CO}_{2}$ do Triple-E é $20 \%$ menor do que da classe antecessora de navios - a E-Class, também da Maersk Line, onde o navio mais conhecido dessa classe foi o Emma Maersk com 15.000 TEUs de capacidade e 397 metros de comprimento (LOA), lançado em 2006; essa eficiência aumenta para 50\% quando compara-se o Triple-E com a média de navios nas rotas da Europa-Ásia; a redução de $37 \%$ no consumo de combustível em virtude de um sistema de recuperação de calor do motor principal e pelo sistema de redução de velocidade (Slow steaming) de 2 motores de 32 Megawatts (43.000 hp) com um sistema de dois hélices a uma velocidade de 19 nós (equivalente a $35 \mathrm{Km} / \mathrm{h}$ ).

Wijnolst e Waals (2000) analisam com base no crescimento do comércio, especialmente no eixo Norte da Europa para o Extremo Oriente e indicam que 2010 seria o ano de introdução dos navios do tipo Malacca-max de ordem de 18.000 TEUs e, de forma simplificada, indicavam cinco Classes de navios, mostrados na tabela 1.

Tabela 1 - Evolução dos navios porta-contêineres em cinco categorias

\begin{tabular}{l|c}
\hline \multicolumn{1}{c|}{ Classes } & Capacidade (em TEUs) \\
\hline Panamax & 4.800 \\
Post-Panamax & 6.600 \\
Maersk S-Class & 8.700 \\
Suez Max & 11.989 \\
Malacca-max & 18.154 \\
\hline
\end{tabular}

Fonte: Wijnolst e Waals (2000)

Ashar (2000) escreve sobre o que ele chamou de uma evolução e quatro revoluções da navegação regular, na qual a evolução refere-se ao crescimento gradual em tamanho dos dois maiores componentes do sistema, isto é, navio e portos; Em seguida, descreve sobre as quatro revoluções, a saber: a primeira revolução é a transferência do navio para o cais (ship-to-shore 
transfer) e a própria invenção do contêiner; a segunda revolução trata da transferência intermodal de navio para a ferrovia (ship-to-rail transfer); a terceira revolução ou revolução do transbordo (transshipment) é a transferência navio para navio (ship-to-ship) e a quarta revolução do estabelecimento de um novo padrão de serviço. Nesse estudo, apresenta-se uma tabela com as dimensões e arranjos de cinco classes de navios, conforme a tabela 2. Semelhante classificação com sete categorias é apresentada na tabela 3.

Tabela 2 - Evolução dos navios porta-contêineres com dimensões e arranjos

\begin{tabular}{l|c|c|c}
\hline Classes & $\begin{array}{c}\text { Capacidade em } \\
\text { TEUs }\end{array}$ & $\begin{array}{c}\text { Dimensóes em } \\
\text { metros }(\text { LOA } \times \text { Boca } \\
x \text { Calado) }\end{array}$ & $\begin{array}{c}\text { Arranjo de Colunas } \\
\text { (convés } x \text { porão } x \\
\text { transversal) }\end{array}$ \\
\hline Panamax & 4.000 & $294 \times 33 \times 12$ & $8 \times 5 \times 13$ \\
Post-Panamax I & 4.000 & $275 \times 39 \times 12,7$ & $8 \times 5 \times 15$ \\
Post Panamax II & 6.000 & $348 \times 42 \times 14$ & $9 \times 6 \times 17$ \\
Double Wide & 15.000 & $400 \times 69 \times 14$ & $10 \times 6 \times 28$ \\
Mallacca-max & 18.000 & $400 \times 60 \times 21$ & $12 \times 8 \times 23$ \\
\hline
\end{tabular}

Fonte: Ashar (2000)

Tabela 3 - Evolução dos navios porta-contêineres com dimensões e arranjos

\begin{tabular}{l|c|c|c}
\hline Classes & $\begin{array}{c}\text { Capacidade em } \\
\text { TEUs }\end{array}$ & $\begin{array}{c}\text { Dimensóes em } \\
\text { metros (LOA x Boca } \\
\text { xCalado) }\end{array}$ & \\
\hline Small Feeder & $<1.000$ & & \\
Feeder & $1.001-2.000$ & & MV Trans Atlantic \\
Feederrmax & $2.001-3.000$ & & Providence Bay \\
Panamax & $3.001-5.100$ & $294 \times 32 \times 12$ & COSCO Guangzhou \\
Post Panamax & $5.101-10.000$ & & Class \\
New Panamax & $10.000-14.500$ & $366 \times 49 \times 15,2$ & E-Class, Triple-E \\
Ultra Large & $>14.501$ & $>366 \times 49 \times 15,2$ & \\
Container Vessel & & & \\
\hline
\end{tabular}

Fonte: DNV offshore containers, 2015

Contudo, a barreira de capacidade de 18.000 TEUs deverá em breve ser superada, está prevista a entrega de um navio de 19.000 TEUs para o armador China Shipping Company Lines 
(CSCL) e, de acordo com pesquisa da empresa de seguros Allianz, a classe de navios de 24.000 TEUs deverá se tornar realidade nos próximos cinco anos.

\section{REVISÃO BIBLIOGRÁFICA - IMPACTOS NOS TERMINAIS DE CONTÊINERES E NAS SUAS OPERAÇÕES}

Como principais itens da lista estão: a questão de aquisição de portêineres que alcancem pelo menos 23 colunas (rows); aumento da capacidade de pátio e não apenas em espaço, mas melhores equipamentos de manuseio com maior capacidade de altura combinados com melhorias dos sistemas de TI ou automação; navios de 400 metros têm se mostrado um desafio para a utilização ótima de berço, em recentes operações de navios como Emma Maersk e CMA CGM Marco Polo demonstram que a combinação do planejamento de janela de tempo (Time windows) com alto grau de acuracidade das escalas não permite otimização de berços. A realidade de aplicação da velocidade reduzida ou inferiores à velocidade de projeto (Slow steaming) melhorou indiretamente a confiabilidade das escalas, pois eventuais atrasos nas operações portuárias podem ser recuperados na navegação acelerando a velocidade.

O aumento das operações de transbordo (Transhipment) acarreta uma maior movimentação interna do terminal, como, por exemplo, o reposicionamento de contêineres nas pilhas e decisões do tipo último-minuto para distribuição dos portos; melhora no processo e sistema de planejamento de navios em virtude do aumento considerável de volume, assim como de operações complexas de conexões com outros modais (serviços de barcaça e trens); ampliação da capacidade de pátio para absorção do volume considerado de descarga, além de espaço excedente para casos de atraso dos navios por: mau tempo ou perda de janela de atracação em portos a montante ou jusante da costa, entre outros (CHOE, KIM, RYU, 2016).

Atenção especial deve ser dada também às áreas de pátio destinadas aos contêineres refrigerados (Reefers), carga perigosa, com excessos (altura, largura e comprimento) e carga geral solta (Caixarias e Carga de projeto); permanece a necessidade de peação e despeação, pois navios sem tampas (Hatchcoverless) continuam raros; de forma geral deverá ser dada mais ênfase às operações ininterruptas por meio de monitoramento constante dos objetivos e metas definidas e uma abordagem industrial na solução de problemas; provavelmente o aumento das demandas de pico deverão exigir equipes de trabalho mais flexíveis; necessidade do aumento da eficiência dos terminais satélites ou de apoio, os quais são conectados operacionalmente com os terminais marítimos e, por derradeiro, o alto rendimento de operações exigirá menos variabilidade de desempenho e não somente nas operações de cais, mas para as operações de transporte interno, 
operação de pátio e processos de gates, com todas as operações físicas suportadas por eventos via EDI para controle logístico. Portanto, um campo fértil para estímulo do aumento da aplicação de automação, ainda que parcial (CHOE, KIM, RYU, 2016).

Contrapondo Rijsenbrij (2001), de forma bastante pragmática, Ward (2012) relata o desafio operacional de movimentar 6.000 movimentos em 24 horas em navios de 15.000 a 18.000 TEUs em cada fluxo do terminal e suas consequências.

O fluxo de navio indica que a produtividade estimada deve ser de 250 movimentos/hora e faz uma relação com o Dwell time típico dos terminais americanos; no fluxo de pátio indica os volumes de transação de gates deve ser de 340 transações/hora para uma operação uniforme de 24 horas, no caso dos gates trabalharem apenas 16 horas esse volume sobe para 560 transações/hora.

Ainda no fluxo de pátio e distribuição de equipamentos, indica um número de 22 RTGs na importação, com produtividade de 20 movimentos/hora para atendimento do navio e outros 10 movimentos/hora para atendimento dos gates, na exportação indica o uso de equipamentos do tipo top-picks (como Reach-stackers) num total de 18 equipamentos, performando 20 movimentos/hora para atender navio ou gates e estabelece uma máquina para cada 100 metros de extensão de quadra e cerca de 400 caminhões circulando ao mesmo tempo para atender às demandas do lado de mar e terra.

Em outro estudo, Rijsenbrij (2002) discorre sobre o decréscimo da capacidade técnica na movimentação de contêineres/hora, de acordo com o tipo do navio, nesse estudo é indicado que quanto maior o arranjo do navio, maiores são as perdas.

Uma comparação dos efeitos entre a operação de navios de 9.000 TEUs versus navios de 18.000 TEUs nos terminais, na condição Ceteris Paribus, isto é, analisando a influência de um fator sobre outro e mantidas as condições inalteradas das variáveis foi realizada por Koegeboehn (2014). A tabela 4 apresenta a comparação.

Tabela 4 - Comparação de uso de recursos entre navios de 9.000 e 18.000 TEUs

\begin{tabular}{l|ccc}
\hline Variável & $\begin{array}{c}\text { Navios } \\
9.000\end{array}$ & $\begin{array}{c}\text { Navios18.000 } \\
\text { TEUs }\end{array}$ & Variação \\
& TEUs & & \\
\hline Pico no pátio & 1,2 & 1,36 & $+13,3 \%$ \\
Slots de pátio & 14.525 & 16.340 & $+12,5 \%$ \\
\hline Portêineres & 5 & 9 & $+80 \%$ \\
\hline & & & $\vdots$ \\
\hline
\end{tabular}




\begin{tabular}{l|ccc}
\hline Transtêineres & 17 & 30 & $+76,5 \%$ \\
Caminhões & 27 & 46 & $+70,4 \%$ \\
Utilização dos portêineres & $59,5 \%$ & $33,1 \%$ & $-44,4 \%$ \\
Utilização dos Transtêineres & $46,1 \%$ & $36,2 \%$ & $-21,5 \%$ \\
Equipe & 1 & 1,47 & $+46,9 \%$ \\
Custo operacional por/contêiner & & & \\
Custo total por/contêiner & 1 & 1,5 & $+50,1 \%$ \\
\hline
\end{tabular}

Fonte: HPC - Hamburg Port Consulting GMbH, 2014

Observa-se que os picos para esses meganavios são maiores, assim como a utilização de recursos, por exemplo: equipamentos de movimentação de cais (portêineres), pátio (transtêineres) e transferência (caminhões e terminal tractors).

Os custos com mão de obra avulsa e própria acompanham a adição desses recursos, mesmo que os volumes de movimentação sejam mantidos; em contrapartida, não há garantias de aumento da eficiência operacional na utilização dos recursos e redução do custo por contêiner.

A variável mais afetada é a necessidade de incremento de portêineres, os quais podem apresentar limitações técnicas, tais como: não alcançar transversalmente determinadas colunas (rows) do navio ou atingir as últimas alturas (layers ou tiers) dos contêineres no convés ou as primeiras alturas do porão (CHOE, KIM, RYU, 2016).

Esses equipamentos possuem vida útil de aproximadamente 15 a 20 anos e estruturas de cais de 50 anos ou mais, o que exige uma longa projeção sobre quando ocorrerá o impacto do tamanho dos navios. Tal evolução dos navios deve ser avaliada no momento da compra ou troca de equipamento, para que não ocorra o subdimensionamento e, consequentemente, restrições de utilização.

Os terminais que possuem Sistemas de Planejamento de Terminal (TOS) podem realizar os sequenciamentos de embarque e descarga de forma automática, por meio de algoritmos que cruzam as informações do plano de bordo e navio (carga em trânsito), contêineres a serem descarregados e posição de estivagem (Stowposition) dos contêineres de embarque, enviado pelo armador a partir de arquivos de Troca Eletrônica de Dados (EDI) MOVINS e BAPLIE com a distribuição e posição dos contêineres no pátio; contudo, é uma função ou módulo do sistema normalmente subutilizado. 
Os quadros 1 e 2 apresentam um resumo bibliográfico sobre planejamento de berços e planejamento e alocação de berços de atracação, respectivamente.

Quadro 1 - Revisão sobre planejamento de berços

\begin{tabular}{|c|c|c|}
\hline $\begin{array}{l}\text { Problemas } \\
\text { Considerados }\end{array}$ & Abordagem & Referências \\
\hline $\begin{array}{l}\text { Planejamento e } \\
\text { Alocação de Berços }\end{array}$ & $\begin{array}{l}\text { Problemas formulados por meio de } \\
\text { programação linear; onde os } \\
\text { problemas menores foram resolvidos } \\
\text { com CPLEX e os casos maiores } \\
\text { foram desenvolvidos com busca tabu } \\
\text { (Tabu search). } \\
\text { Procedimento de busca em árvore } \\
\text { (Tree search). } \\
\text { Experimentos computacionais com } \\
\text { CPLEX, nos quais o primeiro modelo } \\
\text { de DBAP foi capaz de resolver } \\
\text { problemas de } 10 \text { berços e } 50 \text { navios, } \\
\text { enquanto o modelo compacto de } \\
\text { DBAP não foi tão eficiente em } \\
\text { virtude das limitações do grande M's. } \\
\text { Uso de simulação de um sistema } \\
\text { chamado BAMS (Berth Allocation } \\
\text { management System). } \\
\text { Filas de navios atendidas de forma } \\
\text { FIFO. } \\
\text { Modelo de programação inteira mista } \\
\text { (Mixed Integer Program). Algoritmos } \\
\text { Simulated Annealing (SA). } \\
\text { Utilização de gráficos acíclicos } \\
\text { diretos (Directed Acyclic Graph). } \\
\text { Software LINDO, que apresentou } \\
\text { problema de tempo de processamento } \\
\text { quando o número de navios era } \\
\text { superior a sete e o tempo de } \\
\text { planejamento excedia } 72 \text { horas. } \\
\text { Heurística baseada em Algoritmo } \\
\text { Genético (GA). } \\
\text { Programação inteira mista (Mixed } \\
\text { Integer Program) formulando versões } \\
\text { estáticas e dinâmicas do problema de } \\
\text { alocação de berço e suas relaxações } \\
\text { lagrangianas. } \\
\text { Técnicas de otimização subgradiente. }\end{array}$ & $\begin{array}{l}\text { Cordeau et al (2003); } \\
\text { Chen e Hsieh (2001); } \\
\text { Guan e Cheung (2004); } \\
\text { Hansen o Oguz (2003); } \\
\text { Henesey, Davidsson e } \\
\text { Persson (2004); } \\
\text { Imai, Nagaiwa e Chan } \\
\text { (1997); } \\
\text { Kim e Moon (2003); } \\
\text { Lai e Shih (1992); } \\
\text { Legato e Mazza (2000); } \\
\text { Li, Cai e Lee (1998); } \\
\text { Lim (2004); } \\
\text { Lim (2000); } \\
\text { Lim e Goh (2000); } \\
\text { Moon (2000); } \\
\text { Nishimura, Imai e } \\
\text { Papadimitriou (2001); } \\
\text { Park e Kim (2002); } \\
\text { Park e Kim (2003); } \\
\text { Piaw (2003). }\end{array}$ \\
\hline
\end{tabular}

Fonte: Patricio (2005)

Quadro 2 - Revisão sobre planejamento e alocação de berços de atracação

\begin{tabular}{|l|l|l|}
\hline $\begin{array}{l}\text { Problemas } \\
\text { Considerados }\end{array}$ & Abordagem & Referências \\
\hline $\begin{array}{l}\text { Planejamento de } \\
\text { Berço }\end{array}$ & $\begin{array}{l}\text { Teoria das Filas. } \\
\text { Métodos básicos da teoria da }\end{array}$ & $\begin{array}{l}\text { Plumle (1966); } \\
\text { Nicolaou (1967,1969); }\end{array}$ \\
\hline
\end{tabular}




\begin{tabular}{|c|c|c|}
\hline & $\begin{array}{l}\text { probabilidade em sistemas de } \\
\text { portos. } \\
\text { Distribuição discreta e continua } \\
\text { em processos operacionais de } \\
\text { portos. } \\
\text { Processos estocásticos em } \\
\text { sistemas de portos. } \\
\text { Processos Markovianos. } \\
\text { Processos Birth-death. } \\
\text { Processos Poison. }\end{array}$ & $\begin{array}{l}\text { Mettam (1967); } \\
\text { Jones e Blunden (1968); } \\
\text { Miller (1971); } \\
\text { Wanhill (1974); } \\
\text { Edmond (1975); } \\
\text { Noritake e Kimura (1983, } \\
\text { 1989); } \\
\text { Noritake (1985); } \\
\text { Schonfeld e Sharafeldien } \\
\text { (1985); } \\
\text { Sabria e Daganzo (1989); } \\
\text { Radmilović (1992); } \\
\text { Berg-Andreassen e } \\
\text { Prokopowicz (1992); } \\
\text { Huang et al. (1995, 1997); } \\
\text { Zrnić et al. (1999); } \\
\text { Chu e Huang (2002); } \\
\text { Shabayek e Yeung (2001); } \\
\text { Radmilović et al. (2005). }\end{array}$ \\
\hline $\begin{array}{l}\text { Alocação e } \\
\text { Programação } \\
\text { (Scheduling) de } \\
\text { Berço e }\end{array}$ & $\begin{array}{l}\text { Algoritmos DBAP (Dynamic } \\
\text { Berth Allocation Problem). } \\
\text { Algoritmos SBAP DBAP (Static } \\
\text { Berth Allocation Problem). } \\
\text { Algoritmos Genéticos. } \\
\text { Relaxação lagrangiana. } \\
\text { Busca Tabu (Tabu Search). } \\
\text { Programação inteira mista (Mixed } \\
\text { Integer Program). } \\
\text { Procedimento de Busca em árvore } \\
\text { (Tree search). } \\
\text { Simulated Annealing (SA). }\end{array}$ & $\begin{array}{l}\text { Lai e Shih (1992); } \\
\text { Imai et al.(1997); } \\
\text { Lim (1998); } \\
\text { Nishimura et al. (2001); } \\
\text { Imai et al. (2001, 2003, } \\
\text { 2005); } \\
\text { Zhou Xu (2002); } \\
\text { Kim e Moon (2003); } \\
\text { Cordeau et al (2003); } \\
\text { Guan e Cheung (2004). }\end{array}$ \\
\hline
\end{tabular}

Fonte: Dragović e Park (2011)

\section{SISTEMA OPERACIONAL DO TERMINAL - TOS}

O módulo de planejamento de navios é responsável pelo planejamento e sequenciamento de embarque e descarga dos contêineres, visando à redução do número de remoções no pátio; à otimização do posicionamento dos contêineres a bordo; a garantir a estabilidade e cálculo de stress do navio respeitando os dados de características do navio (Vessel Particulars), tais como: limites de peso e altura (Stackweight e Stackheight), e verificação do cumprimento dos cálculos de esforços de peação (lashing) da Lloyds, bem como com os requisitos de peso dinâmico da Lloyds Germanisher a aplicar as informações de pré-estivagem (prestow) do arquivo MOVINS enviado pelo centro de planejamento (planner) do armador, tipo do contêiner, porto de destino, peso do contêiner, posição 
no pátio, instruções especiais de estivagem da carga no porão (Underdeck) ou no convés (Wetherdeck); verificação de conflito de carga perigosa (carga IMDG).

Busk (2009) explica que automação em terminais é primeiramente um TOS inteligente para otimização de recursos por meio de algoritmos avançados e completa que automação está longe de ser apenas equipamentos de movimentação não tripulados.

TOS são softwares de planejamento e gerenciamento operacional de um terminal, sendo basicamente formados por módulos de: Planejamento de Berços; Planejamento de Navio; Planejamento de Pátio; Controle/Alocação de Equipamentos; Controle de Gates; Controle e Registro do Fluxo de documentação; Troca Eletrônica de Dados - EDI; Sistema de Faturamento (Billing) e Interfaces com diversos outros sistemas e dispositivos (CHOE, KIM, RYU, 2016; ZHEN et al., 2012).

Alocação de berço no TOS significa planejar as visitas ou escalas dos navios por meio da reserva de espaço de berço e atribuindo os portêineres antecipadamente de forma a permitir ações preventivas em caso de falta ou avaria. É fato que quase a totalidade dos armadores e terminais trabalha com Janelas Operacionais, o que facilita a programação de berços; mas deve-se lembrar de que a decisão do local de atracação pode implicar o local de abertura do navio o pátio para armazenagem da carga; a definição dos cabeços e a distância dos navios.

\section{AUTOMAÇÃO}

Em 1993 é inaugurado o primeiro terminal de contêineres automatizado da história, o Delta/Sea-Land Terminal (agora chamado de Delta Dedicated North Terminal) da Europe Container Terminal (ECT) do grupo Hutchison Port Holding (HPH) na região de Maasvlakte na Holanda; com o uso de AGVs e ASCs para a transferência de contêineres entre o cais e as pilhas.

Os conceitos e aplicações de automação nas áreas de conhecimento de engenharia são amplos, conforme Pinto (2010) é a tecnologia relacionada com a aplicação de sistemas mecânicos, elétricos, eletrônicos e computacionais nas operações e controle dos sistemas de produção.

Automação é definida como um maquinário que dispõe de dispositivos sensores e de controle, que lhe permitem funcionar automaticamente.

Seleme e Seleme (2008) cunham que automação é distinta da mecanização e explicam que a Mecanização consiste simplesmente no uso de máquina para realizar trabalho em substituição ao esforço físico; enquanto Automação são sistemas automáticos de controle, pelos quais mecanismos verificam seu próprio funcionamento, efetuam medições e correções, sem interferência humana. 
Groove (2011) explica automação como a tecnologia por meio da qual um processo ou procedimento é alcançado sem assistência humana.

Contudo, há um conceito de que é à base do Sistema Toyota de Produção e que não deve ser confundido com a automação, esse conceito é denominado de Autonomação ou automação com um toque humano; normalmente essas máquinas estão acopladas a um dispositivo de parada automático. Segundo Ohno (1997), a Autonomação altera o conceito de gestão, pois não será necessário um operador enquanto a máquina estiver funcionando normalmente, a intervenção humana ocorre apenas em anormalidades ou defeitos.

Shingo (1996) refere-se à Autonomação também como pré-automação e explica que a ideia era de separar completamente os trabalhadores das máquinas por meio do uso de mecanismos sofisticados para detecção de anormalidades na produção. Enquanto Liker (2004) explica que Jidoka é o termo em japonês para Autonomação e significa equipamento dotado de inteligência para desligar-se quando apresenta problemas.

Os sistemas de produção são divididos, segundo Groover (2011), em instalações (a fábrica, os equipamentos e a forma como estão organizados) e Sistemas de Apoio à Produção (conjunto de procedimentos utilizados no suporte da produção e solução de problemas técnicos e logísticos). O mesmo Groover (2011) indica que os elementos automatizados do Sistema de Produção podem ser separados em duas categorias, isto é, automação dos sistemas de produção da fábrica e controle computadorizados dos sistemas de apoio à produção. Todavia, pode-se afirmar que para os processos automatizados de terminais de contêineres e para outros diversos segmentos da indústria, essas duas categorias se sobrepõem, pois a maioria dos sistemas de produção automatizados são suportados e gerenciados por sistemas computacionais.

São três os tipos de automação que classificam os sistemas automatizados de produção. A saber, Automação Rígida; Automação Programável e Automação Flexível.

$\mathrm{Na}$ automação rígida, a configuração do equipamento define a sequência simples das operações de processamento e como características o alto investimento CAPEX inicial em equipamentos; esse item comum aos três tipos de automação, altas taxas de produção e uma inflexibilidade quanto à variação da produção. O processamento dos AGS nos terminais pode ser considerado exemplo de automação rígida.

Enquanto os CLPs dos portêineres são considerados exemplos de automação programável; isto é, equipamento de que são projetados com a capacidade de alterar a sequência de operações de modo a atender a alguma variação. Dentre as características, conforme dito anteriormente, o alto investimento; taxa de produção inferior as taxas da automação rígida; flexibilidade quanto às modificações e maior adaptabilidade para produções em lote (ZHEN et al., 2012). 
Considera-se a automação flexível uma extensão da programável, pois este é capaz de produzir uma variedade de produtos sem grandes desperdícios de produção entre alterações de configurações do equipamento e dos produtos. Como características têm-se: o alto investimento; a produção continua de produtos; taxas médias de produção e alguma flexibilidade para lidar com variações. Por exemplo, os portêineres com os diferentes tipos de contêineres (diversos tamanhos 20", 40", 45"; tipos de contêineres, como plataforma ou flatracks com excesso; cargas de projeto e caixarias).

Há três elementos básicos necessários para automação: energia, sistema de controle e programa de instruções; cada um desses elementos está presente em um dos cinco níveis de automação (BOCK, 2015):

- Nível 1 - do dispositivo

Exemplo: componentes como sensores, atuadores etc.;

- Nível 2 - da máquina

Exemplo: máquinas individuais, tais como: AGVs, Robôs industriais;

- Nível 3 - célula ou do sistema

Trata-se de um grupo de máquinas conectados por um sistema;

- Nível 4 - da fábrica ou da produção

Instruções do sistema de informação corporativo que são traduzidos em planos operacionais para a produção;

- Nível 5 - do empreendimento

Sistema formado pelo sistema de informações corporativo, que inclui funções de gerenciamento da empresa, marketing e vendas, contabilidade, projeto e pesquisa, plano mestre de produção, entre outros.

\section{METODOLOGIA}

A pesquisa é comumente classificada quanto à natureza (básica ou aplicada), à abordagem (qualitativa ou quantitativa), aos objetivos (exploratória, descritiva e explicativa) e aos procedimentos (bibliográfica e documental, de campo e laboratorial ou experimental) (GERHARDT, SILVEIRA, 2009; CIRIBELLI, 2003).

Segundo Vergara (2000) a pesquisa exploratória é utilizada quando há pouco conhecimento sobre o tema abordado. O objetivo da pesquisa exploratória é conhecer com maior profundidade o assunto, tornando-o mais claro. A pesquisa descritiva tem como objetivo descrever as características 
de uma população, fenômeno ou o estabelecimento de relações entre variáveis. Em tal pesquisa o pesquisador não tem papel de interferir e apenas registra, analisa, classifica e interpreta os dados.

Essa pesquisa envolveu um caráter bastante prático das atividades e problemas operacionais e estratégicos de terminais; o que está em linha com as definições de pesquisa segundo Demo (1996) e Minayo (1993). Para Demo pesquisa é uma atividade cotidiana considerada um questionamento ou diálogo permanente com a realidade em sentido teórico e prático; enquanto Minayo (1993) define pesquisa com uma atividade de aproximação sucessiva da realidade que nunca se esgota, combinando teoria e dados (MATTAR, 1999; COOPER; HINDLER, 2003; BABBIE, 2001; SELLTIZ, WRIGHTSMAN, COOK, 1987 e BOTELHO, ZOUAIN, 2006).

Foi levantado um diagnóstico da automação de alguns dos principais terminais de contêineres do porto de Santos, como Brasil Terminal Portuário (BTP); Ecoporto; Embraport; Porto de Itapoá; Santos-Brasil; Tecon Salvador; Tecon Rio Grande; e, Terminal de Vila Velha (TVV).

Do ponto de vista da sua natureza, essa é uma pesquisa aplicada, que de acordo com Silva e Menezes (2001) tem o objetivo de gerar conhecimento para aplicação prática dirigida à solução de problemas específicos.

Observando o ponto de vista da forma de abordagem realizada, o mesmo possui viés de pesquisa qualitativa, quando analisa o processo e seus significados; todavia, possui também uma parcela de pesquisa quantitativa quando classifica e analisa informações através de uso de técnicas estatísticas, tais como: percentagem, média ponderada, etc.

Quanto aos objetivos, é uma pesquisa exploratória, pois visa proporcionar maior familiaridade com o problema com a finalidade de torna-lo explicito ou construir hipóteses, e envolve um levantamento bibliográfico e exemplos que estimulam o entendimento. Possui uma parcela de pesquisa descritiva, quando envolve o uso de técnica de coleta de dados através de questionário e forma de levantamento.

Sobre a metodologia de realização da pesquisa, essa foi realizada através de questionário com perguntas fechadas com escolhas do tipo sim ou não para as aplicações de tecnologia ou dispositivo de automação nos terminais portuários brasileiros.

\section{TECNOLOGIAS UTILIZADAS NOS TERMINAIS BRASILEIROS}

O quadro 3 apresenta um diagnóstico com as tecnologias utilizadas e presentes em oito terminais brasileiros dos portos de Santos (SP), Itapoá (SC), Salvador (BA), Rio Grande (RS), Vila 
Velha (ES), são eles: Brasil Terminal Portuário (BTP); Ecoporto; Embraport; Porto de Itapoá; Santos-Brasil; Tecon Salvador; Tecon Rio Grande e Terminal de Vila Velha (TVV).

Destaca-se que os terminais da BTP e Embraport, no momento da pesquisa em campo possuíam um pouco mais de um ano de operação.

Quadro 3 - Diagnóstico de utilização de tecnologias

\begin{tabular}{|c|c|c|c|c|c|c|c|c|}
\hline & $\frac{8}{8}$ & 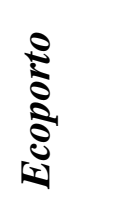 & 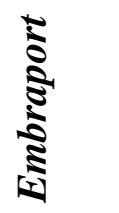 & 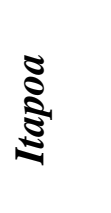 & 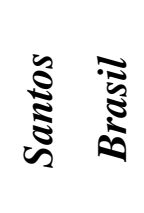 & 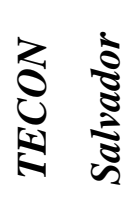 & 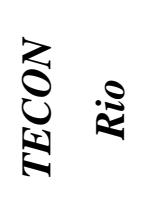 & $\vec{k}$ \\
\hline ACLAS & N/A & N/A & N/A & N/A & N/A & N/A & N/A & N/A \\
\hline AGS & N/A & $X$ & $X$ & N/A & $X$ & N/A & $X(*)$ & $X$ \\
\hline $\mathrm{AGV}$ & N/A & N/A & N/A & N/A & N/A & N/A & N/A & N/A \\
\hline ASC & N/A & N/A & N/A & N/A & N/A & N/A & N/A & N/A \\
\hline A-RTG & N/A & N/A & N/A & N/A & N/A & N/A & N/A & N/A \\
\hline ATHS & N/A & N/A & N/A & N/A & N/A & N/A & N/A & N/A \\
\hline Auto Steering & N/A & $X$ & $X$ & $X$ & $X$ & N/A & N/A & N/A \\
\hline Anti Sway & $X$ & $X$ & $X$ & $\mathrm{X}$ & N/A & N/A & N/A & $X$ \\
\hline Anti Toople & N/A & $X$ & $X$ & N/A & N/A & N/A & N/A & N/A \\
\hline CMS & $X$ & $X$ & $X$ & $X$ & $X$ & N/A & N/A & $X$ \\
\hline EDI & $X$ & $X$ & $X$ & $\mathrm{X}$ & $X$ & $X$ & $\mathrm{X}$ & $X$ \\
\hline OCR (gates) & $X$ & $X$ & $X$ & $X$ & $X$ & $X$ & $\mathrm{X}$ & $X$ \\
\hline OCR (portêineres) & N/A & N/A & N/A & N/A & $X$ & N/A & N/A & N/A \\
\hline Reefer monitoring & N/A & N/A & N/A & N/A & $X$ & N/A & N/A & N/A \\
\hline Remote Control & N/A & $\mathrm{X}$ & N/A & N/A & $\mathrm{X}(\#)$ & N/A & N/A & N/A \\
\hline RFID/RTLS & $X$ & $X(*)$ & $X$ & N/A & $X$ & $X$ & N/A & $X$ \\
\hline SPS & N/A & N/A & N/A & N/A & N/A & N/A & N/A & N/A \\
\hline TPS/VAS & N/A & N/A & $X$ & N/A & N/A & N/A & N/A & N/A \\
\hline TOS & $X$ & $X(*)$ & $X$ & $X$ & $X$ & $X$ & $X$ & $X$ \\
\hline Weigh Scale & $X$ & N/A & N/A & N/A & $X$ & $X$ & $X$ & N/A \\
\hline Eletrificação & N/A & N/A & N/A & N/A & N/A & N/A & N/A & N/A \\
\hline
\end{tabular}

Fonte: Elaborado pelos autores

Legendas: N/A = Não aplicado; X (*) Em fase de implantação; X (\#) Em estudo para RTGs (3 x1)

\section{CONCLUSÃO}


Esse trabalho apresentou através de uma contextualização do setor, a evolução da operação portuária de contêineres e do crescimento de navios, assim como relata esse impacto nas operações e discute a necessidade de adequação permanente dos terminais.

Em seguida, apresenta-se tecnologias e automação aplicada as operações de terminais de contêineres. A literatura internacional, por sua vez, mostrou-se mais ampla com predominância nos tópicos de comparações entre sistemas automatizados de transporte como os AGVs, e de sistemas de armazenamento de pátio automatizado, exemplo dos ASCs (KIM, 2006).

Portanto, foi possível verificar que as operações de terminais são complexas e a automação aumenta essa complexidade, por diversas razões, tal como requerer maior confiabilidade dos sistemas; o custo de CAPEX e/ou OPEX não é desprezível, o impacto na organização do trabalho e layout podem ser um complicador da instalação da tecnologia. E não existe unanimidade sobre a questão automação e qual tecnologia é mais apropriada, porém a tendência observando os 33 terminais automatizados internacionalmente, apenas nove são automação total, os demais tenderam a semi-automação. No caso dos oito terminais brasileiros pesquisados, observou-se que todos possuem o que se classificou com automação menor, com alguns casos incipientes de Sistema de Gate Automatizado (AGS) e uso do TOS.

Pode-se afirmar que a automação no exterior encontra-se em um estágio de controle em respostas às preocupações de elevados custos de mão de obra e necessidade de aumento de produtividade pelo aumento do tamanho dos navios e impacto nos processos do terminal (MARZOUK, ABUBAKR, 2016).

No Brasil, os terminais encontram-se em uma fase de adequação tanto institucional, em virtude do período de incerteza em face dos novos marcos regulatórios, quanto comercial, pela entrada de operadores globais nas operações e excesso temporário de capacidade, e operacional, com o aumento da complexidade da operação pelo crescimento dos navios, e pelas exigências de flexibilidade dos clientes, pelos rígidos controles de segurança, e um ambiente onde não é mais possível, conviver com os gargalos de infraestrutura existente (leia-se acessos terrestres e marítimos).

A questão de automação, encontra-se em uma fase de iniciação, com aplicação e aprendizado de automações menores, conforme demonstrado na pesquisa dos oito terminais, caminhando para uma fase de contágio, de aplicação de automação maior, com risco controlado e de tecnologia com grau de maturidade confiável. 
Em síntese, pode-se sustentar que o cenário para os próximos anos de competição, é que a tendência dos terminais brasileiros é de semi-automação em áreas de gates, planejamento, e de conferência de informações e confirmação de registros.

\section{Referências}

ANTAQ, Agência Nacional de Transporte Aquaviário, 2012.

ASHAR, A. The fourth revolution and transshipment potentials for Panama ports. Terminal Operations Conference (TOC Europa). 16 - 18 May. Rotterdam, 2000.

BABBIE, E. Métodos de pesquisa de survey. Belo Horizonte: Ed. UFMG, 2001.

BOCK, T. The future of construction automation: technological disruption and the upcoming ubiquity of robotics. Automation in Construction, 59, p. 113-121, 2015. crossref

BOTELHO, D.; ZOUAIN, D. M. (Org.). Pesquisa quantitativa em administração. São Paulo: Atlas, 2006.

BUSK, K. Introduction to automation and drivers for automation. Terminal Automation Seminar - Driving terminal efficiency and profitability. London (UK), 2009.

CASACA, A. C. P. Simulation and the lean port environment. Maritime Economics \& Logistics, vol.7.p.262-280, 2005. cross ref

CIRIBELLI, M. C. Como elaborar uma dissertação de mestrado através da pesquisa científica. Rio de Janeiro: Editora 7 Letras, 2003.

CHOE, R.; KIM, J.; RYU, K. R. Online preference learning for adaptive dispatching of AGVs in an automated container terminal. Applied Soft Computing, 38, pp. 647-660, 2016. crossref

COOPER, D.; SCHINDLER, P. Métodos de pesquisa em administração. 7. ed., Porto Alegre: Bookman, 2003.

DAVIDSON, N. Market outlook - Identifying opportunities and trends in the terminal sector. Terminal Management and Planning, Londres, 2014.

DEMO, P. Pesquisa e construção de conhecimento. Rio de Janeiro: Tempo brasileiro, 1996.

DRAGOVIć, B; PARK, N. K. Modeling of ship-berth-yard link performance and throughput evaluation in container port. Masport Seminar: Automation and Simulation Methodologies for the Evaluation and Improvement of Port Container Terminals, 10-11 November, 2011.

DREWRY MARITIME ADVISORS.<http://www.drewry.co.uk/consultancy/>. Acesso em 14 dez. 2014.

GERHARDT, T. E.; SILVEIRA, D. T. (Org.). Métodos de pesquisa. Porto Alegre: Editora da UFRGS, 2009.

GROOVER, M. P. Automação industrial e sistemas de manufatura. São Paulo: Pearson, 2011. 581p.

KIM, J. Y. A TCP/IP-based remote control system for yard cranes in a port container terminal. Robotica, Vol. 24, pp. 613-620, 2006. crossref

KOEGEBOEHN, D. Analysing the development of automation: past, present \& future. Terminal Management and 
Planning. London, 2014.

LIKER, J. K. O modelo Toyota - 14 princípios de gestão do maior fabricante do mundo. Porto Alegre: Bookman, 2004.316p.

MATTAR, F. N. Pesquisa de marketing: metodologia - planejamento. v. 1 e 2, São Paulo: Atlas, 1999.

MARZOUK, M.; ABUBAKR, A. Decision support for tower crane selection with building information models and genetic algorithms. Automation in Construction, 61, p. 1-15, 2016. crossref

MINAYO,M.C.S. O desafio do conhecimento. São Paulo: Hucitec, 1993.

OHNO, T. O sistema Toyota de produção - além da produção em larga escala. Porto Alegre: Bookman, 1997.151p.

PATRICIO, M. Análise de regras de regras de atracação de navios em terminais de contêineres. Dissertação de Mestrado, Escola Politécnica da Universidade de São Paulo (EPUSP). São Paulo, 2005. 127p.

PEIXOTO, G.S.S. Modelo para Seleção de Equipamentos de retaguarda e estratégias de formação de pilhas na armazenagem em terminais de contêineres. Dissertação de Mestrado, Escola Politécnica da Universidade de São Paulo (EPUSP). São Paulo, 2005. 151p.

PINTO, J. R. C. Técnicas de automação. Lisboa (Portugal): ETEP, 2010.420p.

RIJSENBRIJ, J. C. The impact of tomorrow's ships on landside. Terminal Operations Conference (TOC-Europa), 19-21 June.Lisbon, 2001.

ROBINSON, H.J. Formação de estoques de contêineres em áreas portuárias. Dissertação de Mestrado, Instituto Militar de Engenharia (IME). Rio de Janeiro, 1985. 288p.

SALGADO, M. Terminais de contêineres: Investir continuamente: questão de sobrevivência. $\mathbf{1}^{\circ}$ Fórum de Contêineres: Investimentos em Terminais de Contêineres: Apostando no Processo de Industrialização Nacional. São Paulo, 2012.

SELEME, R; SELEME, R. B. Automação da produção - abordagem gerencial. Paraná: Editora IBEX, 2008. 215p.

SELLTIZ, C.; WRIGHTSMAN, L.; COOK, S. Métodos de pesquisa nas relações sociais. São Paulo: EPU, 1987.

SHINGO, S. O sistema Toyota de produção do ponto de vista da engenharia de produção. Porto Alegre: Bookman, 1996.

SILVA, E. L.; MENEZES, E. M. Metodologia da pesquisa e elaboração de dissertação. Florianópolis: Laboratório de Ensino a distância da UFSC, 2001. 121p.

STEENKEN, D.; VO $\beta$, S.; STAHLBOCK, R. Container terminal operation and operations research - a classification and literature review. OR Spectrum, v.26, p.3-49, 2004. crossref

VERGARA, S. C. Projetos e relatórios de pesquisa em administração. São Paulo: Atlas, 2000.

ZHEN, L.; LEE, L. H..; CHEW, E. P.; CHANG, D-F; XU, Z-X. A Comparative study on two types of automated container terminal systems. IEEE Transactions on Automation Science and Engineering, vol. 9, no. 1, January, 2012. cross ref

WARD, T. Terminal efficiency - expectations and responses. Terminal Operations Conference (TOC Europe), $13^{\text {th }}-$ $15^{\text {th }}$ June. Antwepen, 2012. 
WIJNOLST, N.; WAALS, F. Malacca-max container carrier on the Europe-far east route by 2010. Terminal Operations Conference (TOC Europe), 16 - 18 May, Rotterdam, 2000.

\section{Dados dos autores:}

\section{Nome completo: Delmo Alves de Moura}

Filiação institucional: Universidade Federal do ABC - UFABC

Departamento: Centro de Engenharia, Modelagem e Ciências Sociais Aplicadas

Função ou cargo ocupado: Professor Adjunto IV

Endereço completo para correspondência: Avenida dos Estados, 5001 - Santo André - SP - Brasil

Telefones para contato: (11) 4996-8284

E-mail:delmo.moura@ufabc.edu.br

Nome Completo: Marclo Patrício

Filiação institucional: Brasil Terminal Portuário

Função: Gerente Geral de Operações

Endereço: Av. Engenheiro Augusto Barata s/n - CEP 11095-907, Alemoa, Santos, SP, Brasil

Telefones para contato: (13) 3229-4040

E-mail:m.patricio@btp.com.br

Nome Completo: Rui Carlos Botter

Filiação institucional: Universidade de São Paulo - USP

Departamento de Engenharia Naval e Oceânica

Escola Politécnica da Universidade de São Paulo - POLI

Função: Profesor Titular

Endereço: Av. Prof. Mello Moraes, 2231. CEP 05508-030 - São Paulo- SP - Brasil

Telefones para contato: (11) 3091-1724

E-mail:rcbotter@usp.br

Submetido em:04-10-2016

Aceito em: $31 / 12 / 2016$ 